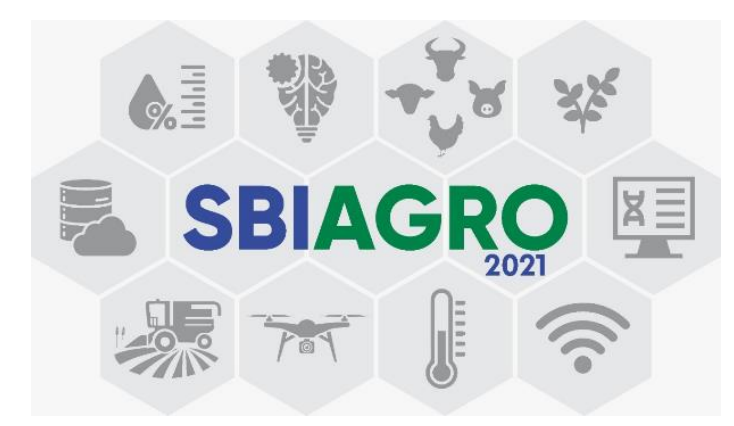

\title{
Controle de temperatura e de umidade de uma composteira utilizando o ESP32
}

\author{
Luan D. Rodrigues ${ }^{1}$, Rodrigo M. Pereira ${ }^{1}$, David Willy F. Palmera ${ }^{1}$, Guilherme \\ Henrique A. Silva1, Marco Antonio F. Leite ${ }^{1}$, Juliana S. Silva ${ }^{2}$ \\ ${ }^{1}$ Departamento de Área de Eletroeletrônica, Campus Cuiabá - Cel. Octayde Jorge da \\ Silva, Instituto Federal do Mato Grosso, Cuiabá, Mato Grosso, Brasil. \\ ${ }^{2}$ Departamento de Área de Computação, Campus Cuiabá - Cel. Octayde Jorge da Silva, \\ Instituto Federal do Mato Grosso, Cuiabá, Mato Grosso, Brasil.

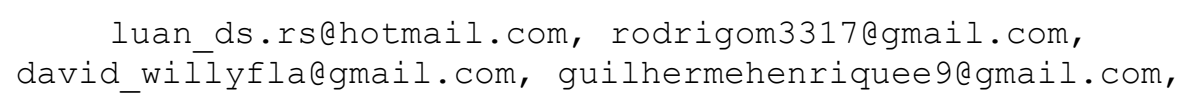

\begin{abstract}
The Organic Agriculture has become applicable to several crops, due to its low environmental impact, since it does not use chemical inputs and genetically modified organisms in cultivation, as occurs, in general, in conventional agriculture, which is widely spread in Brazil. However, it is noteworthy that there are difficulties in the practice of organic agriculture, since the microorganisms responsible for the decomposition process of organic matter need a suitable environment for their proliferation. Therefore, the objective of this article is to present the creation of a composter prototype, with automatic temperature and humidity control. Thus, for the development of the prototype, the following software were used: (i) Arduino IDE; (ii) Fritzing; and (iii) Inventor. The hardware components were: (i) ESP32 microcontroller; (ii) Humidity sensor; (iii) Stepper motor; and (iv) Driver A4988. With the combination of hardware and software resources, it was possible to build the prototype. The results were satisfactory, because the composter structure was controlled through the analysis of information measured by sensors, using ESP32.
\end{abstract}

Resumo. A Agricultura Orgânica vem tornando-se aplicável em diversos cultivos, em razão do seu baixo impacto ambiental, pois em seu cultivo não há a utilização de insumos químicos e organismos geneticamente modificados, como ocorre, em geral, na agricultura convencional que é amplamente difundida no Brasil. No entanto, vale ressaltar que existem dificuldades para a prática da agricultura orgânica, visto que os microrganismos responsáveis pelo processo de decomposição da matéria orgânica necessitam de um meio propício à sua proliferação. Portanto, o objetivo deste artigo é apresentar a 
criação de um protótipo de uma composteira, com controle automático de temperatura e umidade. Assim, para o desenvolvimento do protótipo, foram utilizados os seguintes software: (i) Arduino IDE; (ii) Fritzing; e (iii) Inventor. Os componentes de hardware foram: (i) Microcontrolador ESP32; (ii) Sensor de umidade; (iii) Motor de passo; e (iv) Driver A4988. Com a junção dos recursos de hardware e software foi possivel confeccionar o protótipo. Os resultados alcançados foram satisfatórios, pois a estrutura da composteira foi controlada por meio da análise das informações medidas pelos sensores, utilizando o ESP32.

\section{Introdução}

Entre os séculos XVIII e XIX, ocorreu a revolução agrícola, trazendo como elementos basilares técnicas modernas, como o uso de fertilizantes, agrotóxicos, organismos geneticamente modificados e a monocultura - que contribuíram para o aumento da produção e a redução de custos [Bin 2004]. No entanto, isso ocasionou uma série de impactos ambientais, tais como, degradação do solo, contaminação da água, intoxicação de agricultores e aparecimento de novas pragas. Levando em consideração todos esses problemas, surgiu o conceito de uma agricultura mais sustentável, sendo denominada de Agricultura Orgânica [São Paulo 2011].

Foi justamente nesse contexto que, no decorrer da década de 1940, o pesquisador inglês Albert Howard, durante pesquisas em viagens à Índia, examinou a utilização de meios orgânicos na agricultura, a partir do uso de práticas de adubação orgânica. De forma paralela, outros pesquisadores também realizaram estudos sobre a implementação da agricultura biológica, com escasso uso de insumos agroquímicos [Ormond et al. 2014]. Com isso, a Agricultura Orgânica nasceu como uma forma de manejo voltada a mitigar os impactos ao meio ambiente, pois se utiliza de insumos de origem orgânica, gerados por meio de técnicas como a compostagem; prevenindo, assim, o uso de agrotóxicos e organismos geneticamente modificados [Alves, Santos e Azevedo 2012].

A compostagem é um método de decomposição da matéria orgânica de forma controlada. Apesar dos benefícios advindos do seu uso, como baixo custo e menor impacto ambiental, é importante lembrar que consiste em um método de decomposição da matéria difícil de ser realizado, uma vez que há a necessidade da intervenção de microrganismos decompositores, que dependem de um meio favorável à sua sobrevivência. Por conseguinte, diversos fatores precisam ser controlados para que a compostagem gere um insumo desejável - como a temperatura, a umidade, a aeração, o pH do solo, a granulometria e a relação Carbono e Nitrogênio (C/N) [Valente et al. 2009]. Alguns desses fatores (como a temperatura e a umidade) podem ter um controle automático, o que pode contribuir para a redução no tempo de trabalho do produtor rural. Caso o controle ocorra de forma adequada, o processo da compostagem tende a ser otimizado.

Diante desta conjuntura, surge uma oportunidade para se realizar diversas pesquisas a respeito dessa temática. Sendo assim, o objetivo deste artigo é apresentar a criação de um protótipo de uma composteira, com controle automático de temperatura e umidade, utilizando o ESP32. 
Desta forma, o artigo está dividido em cinco seções, incluindo esta introdução. A seção 2 traz os conceitos teóricos basilares para a compreensão acerca dos temas trabalhados no decorrer da pesquisa. A seção 3 apresenta os materiais e métodos utilizados no desenvolvimento do protótipo. Na sequência, a seção 4 aborda o processo de construção da composteira. Por fim, a seção 5 explicita as principais vantagens e dificuldades para a elaboração deste protótipo.

\section{Revisão Bibliográfica}

Esta seção tem como objetivo descrever, de forma sucinta, os termos fundamentais para a compreensão desta pesquisa, como Agricultura Orgânica, Compostagem e Dispositivos Computacionais.

\subsection{Agricultura Orgânica}

A Agricultura Orgânica consiste na produção de alimentos utilizando os recursos de matéria orgânica presentes no solo, sem a adição de fertilizantes químicos artificiais, que são necessários para o desenvolvimento do cultivo agrícola. A presença desse material no solo é resultado do trabalho dos microrganismos já estabelecidos nos compostos biodegradáveis [Ormond et al. 2014].

Essa prática auxilia na diminuição da degradação ao meio ambiente, visto que não há a utilização de agrotóxicos ou a alteração nas características genéticas do cultivável [Alves, Santos e Azevedo 2012]. No entanto, quando se utiliza de agrotóxicos, observamse diversos impactos negativos (tais como: erosão, intoxicação do solo e poluição da água), tanto para a saúde dos agricultores, quanto para os que consomem esses produtos [Souza 2005]. Em função dessa realidade, nota-se uma preocupação crescente com o meio ambiente, a saúde e o bem estar humano - fatores que têm contribuído para o incremento do mercado de produtos orgânicos no Brasil [Barbosa e Sousa 2012].

É importante mencionar, ainda, que existem diversas estratégias para a produção de matéria orgânica, a ser utilizada nas plantações, dentre as quais a compostagem é um exemplo - como descrito a seguir.

\subsection{Compostagem}

A compostagem consiste em um processo de decomposição orgânica, gerado a partir de ações de um conjunto diversificado de microrganismos (de forma aeróbica ou anaeróbica), que são realizadas em uma série de etapas, de forma sequencial, que são divididas em dois grupos, sendo eles: termofílica e mesofílico. No meio deste processo, várias ações favorecem condições termofílicas, gerando calor, que é dissipado em todo o composto [Oliveira, Sartori e Garcez 2008].

Assim, o objetivo principal da compostagem é gerar um produto que possa ser utilizado como adubo orgânico, que tem um alto valor químico-físico-biológico [Pires e Ferrão 2017]. Um exemplo de uma composteira manual, frequentemente utilizada em residências, onde o indivíduo deve realizar o manuseio do composto com as próprias mãos, sendo disposto na Figura 1. 


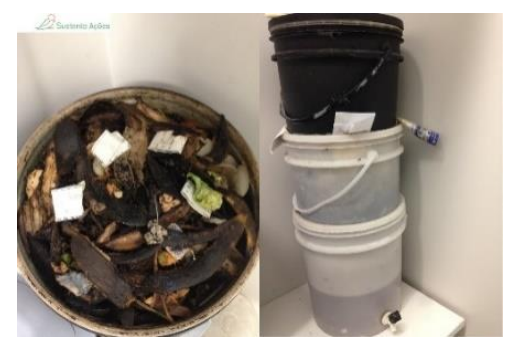

Figura 1. Composteira em balde [Lobe 2019]

Para que o processo de compostagem seja eficiente, o composto precisa estar propício à vida, ou seja, deve estar bem aerado, possibilitando a presença de oxigênio.

Desta forma, as temperaturas, dentro de uma composteira, devem passar dos $50^{\circ} \mathrm{C}$ (para eliminar agentes patológicos), mas não exceder à $70^{\circ} \mathrm{C}$ - pois, assim, a vida dos microrganismos será afetada. Ademais, a umidade no meio do composto deve estar entre $40 \%$ e $60 \%$, pois, abaixo desse valor, a atividade microbiana pode ser inibida e, acima, gera condição de anaerobiose (ações geradas por organismos que não usam oxigênio) [Valente et al. 2009].

Além disso, para facilitar o monitoramento e o controle das variáveis umidade e temperatura, pode-se utilizar de dispositivos computacionais de apoio, como sensores e microcontroladores - como descrito a seguir.

\subsection{Dispositivos Computacionais}

Microcontrolador é um dispositivo computacional [Oliveira Júnior e Duarte 2010], constituído pela junção de diversos componentes, em um único chip: um processador, portas de entrada/saída, memória de acesso aleatório (Random Access Memory - RAM) e memória somente de leitura (Read Only Memory - ROM) [Silva, Targa e Cezar, 2019].

Existem diversas aplicações dos microcontroladores, destacando-se a Automação Residencial e a Agricultura de Precisão [Maia 2012]. Para realização dessas atividades, são desenvolvidas programações específicas, utilizando-se, em geral, as linguagens Python ou $C$.

Dentre os diversos modelos de microcontroladores disponíveis no mercado e de ampla utilização em pesquisas estão: o Arduino $^{1}$ e o $\operatorname{ESP}^{2} 2^{2}$ [Pereira 2020]. Para desenvolver o projeto em questão, utilizou-se o ESP32, por possuir uma maior conectividade com a rede externa (internet).

Ademais, para realizar o monitoramento de diversas variáveis do ambiente (como umidade, temperatura, índice pluviométrico, incidência solar, acidez do solo, entre outros), torna-se necessário utilizar alguns sensores.

Os sensores são equipamentos que podem receber estímulos do ambiente, sendo sensíveis à luminosidade, energia cinética e térmica. Com isso, têm a capacidade de transformar esses estímulos em valores de grandezas conhecidas (tais como: temperatura, pressão, velocidade, corrente elétrica, aceleração e posição), em um sinal de saída

\footnotetext{
${ }^{1}$ Informações adicionais sobre a placa microcontrolada Arduino estão disponíveis no site do fabricante: https://www.arduino.cc

2 Detalhes a respeito da placa microcontrolada ESP32 podem ser consultadas no Datasheet: https://www.espressif.com/sites/default/files/documentation/esp32_datasheet_en.pdf.
} 
[Wendling 2010]. Existem dois tipos de sensores, o analógico e o digital, sendo separados pelo tipo de sinal de saída [Thomazini e Albuquerque 2011] - ambos foram utilizados no desenvolvimento do protótipo.

Visto que alguns conceitos essenciais para o trabalho foram expostos, a seção seguinte dedica-se à apresentação do material e métodos utilizados na criação do protótipo.

\section{Material e Metódos}

O presente trabalho tem como objetivo a elaboração de um protótipo para realizar o controle de temperatura e umidade de uma composteira utilizando o microcontrolador ESP32.

Para tanto, o desenvolvimento deste trabalho compreendeu às seguintes etapas: (i) Revisão da literatura; (ii) Identificação dos problemas enfrentados para o pleno funcionamento de uma composteira; (iii) Desenvolvimento do protótipo de uma composteira, utilizando recursos de hardware e software; e (iv) Teste e análise dos resultados.

$\mathrm{Na}$ etapa (i) houve a busca por artigos na área, com o propósito de apresentar conceitos básicos para facilitar o entendimento do tema. Na sequência, a etapa (ii) consistiu no mapeamento dos recursos de hardware e software necessários para o desenvolvimento do protótipo, analisando o custo-benefício gerado, para o proprietário, com a implementação do equipamento.

Com a escolha dos equipamentos que seriam utilizados, a etapa (iii) compreendeu a criação do protótipo para o controle de temperatura e umidade de uma composteira, utilizando o microcontrolador ESP32. Assim, foi realizado a programação e a simulação do protótipo, utilizando-se os recursos de software e de hardware, dispostos, respectivamente, nos Quadros 1 e 2.

Quadro 1. Softwares utilizados

\begin{tabular}{|cll|}
\hline Software & \multicolumn{1}{c|}{ Definição } & Figura \\
\hline \multirow{5}{*}{ Arduino IDE } & $\begin{array}{l}\text { Ambiente de } \\
\text { desenvolvimento } \\
\text { integrado, utilizado para a } \\
\text { implementação do código } \\
\text { de programação. A versão } \\
\text { adotada foi a 1.8.9. }\end{array}$ \\
& $\begin{array}{l}\text { Ambiente utilizado para } \\
\text { realizar a simulação dos } \\
\text { circuitos necessários do } \\
\text { protótipo; versão } \\
\text { empregada - 0.9.3b.64. }\end{array}$ \\
& \\
Inventor & fritzing \\
& $\begin{array}{l}\text { Possibilitou a criação da } \\
\text { estrutura virtual do } \\
\text { protótipo; versão utilizada } \\
\text { - Autodesk Inventor } \\
\text { 2019. }\end{array}$ \\
\end{tabular}

Fonte: elaborado pelos autores
Quadro 2. Componentes de Hardware utilizados

\begin{tabular}{|ll|}
\hline Hardware & \multicolumn{1}{c|}{ Definição } \\
ESP32 & $\begin{array}{l}\text { Consiste em um } \\
\text { microcontrolador, que foi } \\
\text { utilizado para receber o } \\
\text { código do compilador, } \\
\text { para realizar o } \\
\text { acionamento e a leitura } \\
\text { dos dados da } \\
\text { composteira. }\end{array}$ \\
Sensor de & $\begin{array}{l}\text { Sensor de umidade } \\
\text { capacitivo analógico, } \\
\text { utilizado para a medição } \\
\text { da umidade do solo. }\end{array}$ \\
Motor de & $\begin{array}{l}\text { Motor com precisão de } \\
\text { posicionamento. }\end{array}$ \\
& \\
Passo & \\
Driver utilizado para \\
controlar a rotação do \\
motor de passos.
\end{tabular}

Fonte: elaborado pelos autores 
A última etapa desta pesquisa (iv), consistiu na elaboração de testes e na análise dos resultados, por meio dos quais foi possível identificar os principais benefícios da implementação do protótipo.

Após a apresentação das etapas e dos recursos de software e hardware utilizados nesta pesquisa, a próxima seção tem por objetivo descrever o processo de desenvolvimento do protótipo da composteira.

\section{Protótipo da composteira automatizada}

As principais etapas a serem considerados para a criação de uma composteira, com controle automático de umidade e temperatura, estão descritas a seguir.

\subsection{Etapa 1: Simulação dos Circuitos}

Esta etapa consistiu na simulação dos circuitos necessários para a construção do protótipo, por meio do programa Fritzing. Na simulação realizada (Figura 2), onde foram conectados todos os dispositivos utilizados para montagem do protótipo, juntamente com a alimentação necessária. Com isso, foi possível estabelecer os testes dos circuitos de monitoramento de umidade, temperatura e rotação do recipiente da composteira.

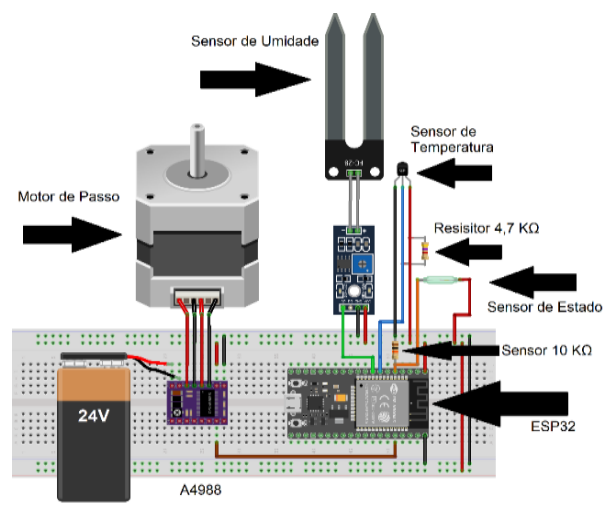

Figura 2. Montagem Virtual do Circuito utilizado no protótipo da composteira

\subsection{Etapa 2: Desenvolvimento da programação}

Esta etapa compreendeu a criação do código fonte responsável pelo controle das variáveis temperatura e umidade, bem como da rotação do motor. A programação deste código fonte foi realizada no Software Arduino IDE, utilizando a linguagem $C$.

Como requisito para o desenvolvimento deste código fonte, foi definido que a rotação do balde da composteira seria constituída por um motor, que foi iniciado, automaticamente, sempre que os valores dos parâmetros de umidade e temperatura excedessem um valor estipulado no código fonte. Ademais, as variáveis de temperatura e umidade foram mensuradas, periodicamente, por meio do sensor DS18B20 e do Módulo Sensor de Umidade para Solos.

É importante registrar que a versão final do código desenvolvido e a esquemática para a criação do projeto estão disponíveis para a visualização no site do GitHub, na seguinte $u r l$ : https://github.com/Luan-DR/Controle_Automatico_de_Uma_Composteira. 


\subsection{Etapa 3: Estrutura da composteira}

$\mathrm{Na}$ terceira etapa, houve a elaboração da modelagem de uma estrutura da composteira, por meio do software Inventor, o que possibilitou a visualização do modelo 3D (Figura 3), para a construção da composteira.

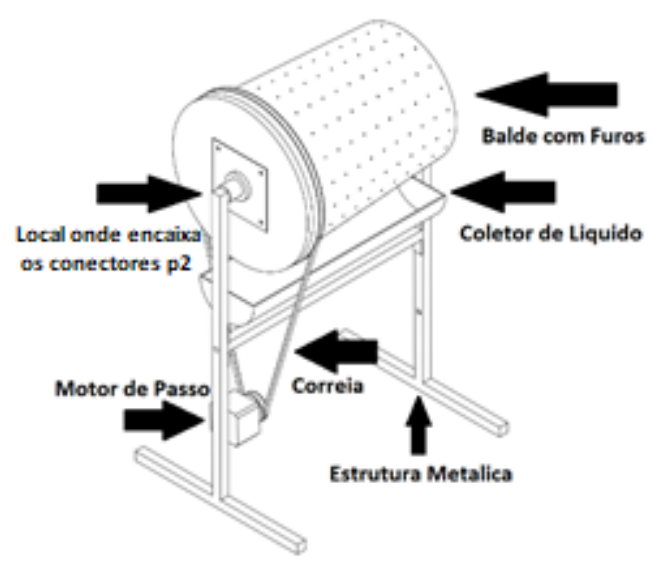

Figura 3. Modelo 3D da Composteira confeccionado no software Inventor

Uma vez que foram explicitados os passos utilizados para a montagem do protótipo, a próxima seção se dedica a abordar o funcionamento do equipamento desenvolvido.

\section{Resultados e Discussão}

Como resultado deste trabalho, foi possível construir o protótipo final da composteira, como demonstrado na Figura 4. Neste protótipo, o sensor de temperatura DS18B20 (disposto na Figura 4a) realizou sua comunicação por meio do protocolo One Wire, que, para sua utilização, necessitou das seguintes bibliotecas: OneWire.h e DallasTemperature. Estas bibliotecas têm a finalidade de interpretar os valores obtidos pelo sensor de temperatura.

Além disso, o sensor de umidade (também disposto na Figura 4a) realizou a leitura de dados analógicos, cujas tensões variaram entre $0 \mathrm{~V}$ e 3,3V. Assim, para que o usuário conseguisse manuseá-lo foi necessário realizar uma interpolação dos valores dos sinais, que relacionou o valor analógico, aferido no sensor, com a porcentagem de água presente no composto.
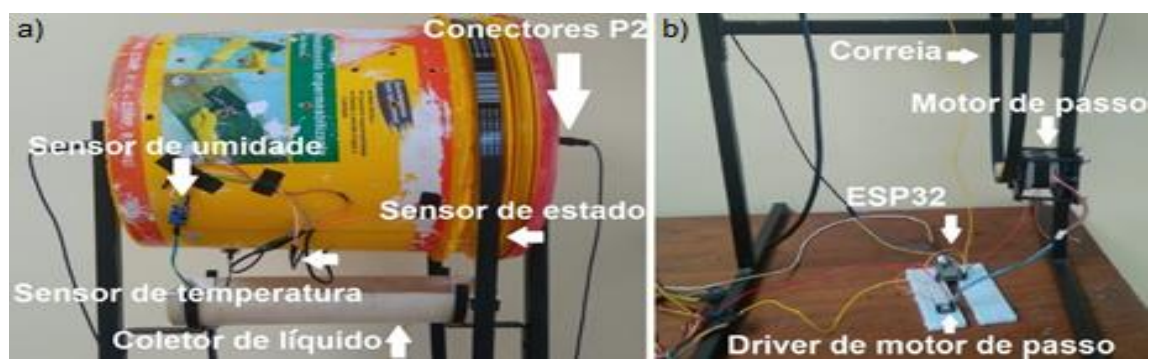

Figura 4. Protótipo final da composteira

Já a utilização dos conectores P2 (Figura 4a), justificou-se por ser uma forma simples para o transporte de dados dos sensores para o microcontrolador e, assim, 
possibilitou a rotação em torno de um eixo, mesmo com o ESP32 estando fora do balde (Figura $4 \mathrm{~b}$ ), o que facilitou sua alimentação e o controle do motor.

É importante salientar, também, que a estrutura da composteira necessita ser de um material resistente, para suportar o balde repleto de composto orgânico; por esse motivo, a estrutura do protótipo foi construída de ferro, que foi movimentada por um motor de passo, de 8 a $32 \mathrm{~V}$, alimentado por corrente contínua (como pode ser visualizado na Figura 4b).

Por fim, com relação à literatura disponível, faz-se necessário registrar que existem trabalhos semelhantes a este, como o de [Silva 2019], que se realizou um monitoramento de temperatura, umidade e oxigênio; porém, o trabalho não se aplicou um microcontrolador. Ademais, para o armazenamento do composto, o autor utilizou leiras estáticas (método de compostagem feito no solo), enquanto, no projeto aqui proposto, o monitoramento foi realizado em um balde, possibilitando, assim, a implantação desse protótipo em pequenas residências.

\section{Conclusão}

Por meio do desenvolvimento deste trabalho, pode-se concluir que a implementação do protótipo ocorreu de maneira satisfatória, uma vez que, foi possível realizar o controle automatizado de um composto orgânico por meio dos dados mensurados pelos sensores de umidade e de temperatura. Face ao exposto, salienta-se a importância da utilização de um protótipo como este para a produção de matéria orgânica, como viabilidade para a aplicação nos cultiváveis.

Na elaboração do trabalho surgiram algumas dificuldades, sendo, a primeira, a montagem da estrutura metálica da composteira, em função de sua capacidade de armazenamento que era de 20L. A outra consistiu no posicionamento correto dos sensores, visando a melhor veracidade dos dados coletados.

Além disso, tem-se a perspectiva da realização de trabalhos futuros, a partir deste protótipo, onde será desenvolvido um aplicativo e um website, possibilitando uma interação com o usuário final. Com isso, existe a necessidade de um servidor próprio para armazenar os dados, para que os resultados do monitoramento da composteira sejam mostrados ao usuário por meio de gráficos e relatórios.

\section{Referências}

Alves, A. C. O., Santos, A. L. S. e Azevedo, R. M. C. (2012) "Agricultura orgânica no Brasil: sua trajetória para a certificação compulsória”. Revista Brasileira de Agroecologia. [S. I.], vol. 7, n. 2, pp 19-27, abr. 2012.

Barbosa, W. F. e Sousa, E. P. (2012) "Agricultura orgânica no Brasil: características e desafios”. Revista Economia \& Tecnologia, [S.I.], v. 8, n. 4, p. 67-74, 2012.

Bin, A. (2004) Agricultura e meio ambiente: contexto e iniciativas da pesquisa pública Campinas, SP, 2004.

Lobe, L. M. K. (2019) Benefícios de um minhocário ou composteira - Meu santo composto. Disponível em: http://www.sustentaacoes.com/2019/02/beneficios-de-umminhocario-ou-composteira-meu-santo-composto-1.html. Acesso em: 17 out 2020. 
Maia, G. (2012) Acionamento Remoto de Portões Elétricos Via Celular Através de Microcontrolador. Orientador: Profa. M. C. Maria Marony Sousa Farias. 1 f. Trabalho de Conclusão de Curso (Engenharia de Computação) - Centro Universitário de Brasília, Brasília.

Oliveira, E. C. A., Sartori, R. H. e Garcez, T. B. Compostagem. (2008) 19 p. Universidade de São Paulo - Escola Superior de Agricultura Luiz Quieroz, Piracicaba, 2008. Disponível em: https://www.agencia.cnptia.embrapa.br/Repositorio/Compostagem_000fhc8nfqz02w yiv80efhb2adn37yaw.pdf. Acesso em: 13 out. 2020.

Oliveira Júnior, M. e Duarte, R. O. (2010) Introdução ao Projeto com Microcontroladores e Programação de Periféricos. Universidade de Minas Gerais. (Apostila de Introdução ao Projeto com Microcontroladores e Programação de Periféricos).

Ormond, J.G.P., Paula, S. R. L., Faveret Filho, P. S. C. e Rocha, L. T. M. (2014) Agricultura Orgânica: Quando o Passado é Futuro. Biblioteca Digital do BNDES, BNDES - Biblioteca Digital, 17 out. 2014. Disponível em: https://web.bndes.gov.br/bib/jspui/handle/1408/2479. Acesso em: 10 nov. 2020.

Pereira, C. L. O. (2020) Programação de Microcontroladores para a Comunicação com Aplicações Móveis Usando Bluetooth. 44 f. Trabalho de Conclusão de Curso (Engenharia da Computação) - Pontifícia Universidade Católica do Rio de Janeiro, Rio de Janeiro.

Pires, I. C. G e Ferrão, G. E. (2017) "Compostagem no Brasil sob a perspectiva da legislação ambiental”. Revista Trópica: Ciências Agrárias e Biológica, [S. I.], v. 9, n. 1, pp. 1-18.

São Paulo (Estado). (2011) Secretaria do Meio Ambiente / Coordenadoria de Biodiversidade e Recursos Naturais. Agricultura sustentável. Kamiyama, Araci. São Paulo: SMA. 75p., 21 x 29,7cm (Cadernos de Educação Ambiental, 13).

Silva, J. R. (2019) Automação do Processo de Compostagem: uso de sensores para monitoramento e controle de parâmetros de um processo sustentável. 2019. 75 p. Dissertação (Mestrado em Engenharia de Produção) - Universidade Federal de Goiás, Catalão.

Silva, M. C., Targa, (2019) M. S. e Cezar, V. R. S. Uso de Microcontrolador Arduino para a Determinação da Permeabilidade do Solo [S. 1.], p. 1-28.

Souza, J. (2005) Agricultura Orgânica: Tecnologias para a Produção de Alimentos Saudáveis. -Instituto Capixaba de Pesquisa. v. 2, 257 p.

Thomazini, D. e Albuquerque, P. U. B. (2011) Introdução. In: Sensores Industriais: Fundamentos e Aplicações. 4. ed. rev. São Paulo: Érica.

Valente, B. S. et al .(2009) "Fatores Que Afetam O Desenvolvimento Da Compostagem De Resíduos Orgânicos”. Archivos de Zootecnia, [S. I.], vol. 58, pp. 59-85, maio.

Wendling, M. (2010) Sensores. Apostila da Universidade Estadual Paulista. 19 p. Disponível

em: https://www.feg.unesp.br/Home/PaginasPessoais/ProfMarceloWendling/4--sensores-v2.0.pdf. Acesso em: 25 maio 2021. 